\title{
Autoestima, empatía y conducta agresiva en adolescentes víctimas de bullying presencial
}

\author{
Maite Garaigordobil, Vanesa Martínez-Valderrey y Jone Aliri \\ Universidad del País Vasco (España)
}

\begin{abstract}
Los problemas de convivencia escolar están muy presentes en las aulas, y no sólo dificultan el desarrollo de los procesos de enseñanza-aprendizaje, sino que además tienen consecuencias nefastas para el desarrollo humano. Con esta contextualización, el estudio tuvo tres objetivos: 1) Analizar si existen diferencias en función del género y el nivel socio-económico-cultural en victimización; 2) Estudiar las relaciones entre victimización y otras variables como autoestima, empatía, y agresividad, explorando si existen diferencias entre víctimas y no-víctimas en estas variables; e 3) Identificar variables predictoras de victimización, es decir, de ser víctima de bullying "cara a cara". La muestra se configura con 178 participantes de 13 a 15 años. Se utilizó un diseño correlacional, administrando cuatro instrumentos de evaluación. Los resultados confirmaron que el nivel de victimización en varones y mujeres fue similar. Además, se constató que en los tres niveles socio-económico-culturales (bajo-medio-alto) la victimización fue análoga. Los adolescentes de ambos sexos con altas puntuaciones en victimización tuvieron significativamente menor nivel de autoestima. Sin embargo, no se hallaron correlaciones entre victimización (ser víctima de bullying "cara a cara") y empatía, ni tampoco entre victimización y agresividad (ni impulsiva, ni premeditada). Además, en el análisis de regresión dos variables resultaron predictoras de victimización: baja autoestima y menor nivel de edad.
\end{abstract}

Palabras clave: Bullying, autoestima, empatía, agresividad.

Self-esteem, empathy and aggressive behavior in adolescent victims of bullying "face-toface". Problems of coexistence at school are very obvious in the classroom, not only hindering the progress of teaching-learning processes but also having nefarious consequences for human development. Within this context, the study had three goals: 1) To analyze possible differences in victimization as a function of gender and socioeconomic-cultural level; 2) To study the relations between victimization and other variables such as self-esteem, empathy, and aggressiveness, exploring possible differences between victims and non-victims in these variables; and 3) To identify predictors of victimization, that is, being a victim of "face-to-face" bullying. The sample is made up of 178 participants aged between 13 and 15 years. A correlational design was used, administering four assessment instruments. Results confirmed a similar level of victimization in males and females. Moreover, analogous victimization was confirmed in the three socio-economic-cultural levels (low-medium-high). Adolescents of both sexes scoring high in victimization had significantly lower levels of self-esteem. However, no significant correlations were found between victimization (i.e., being a victim of "face-toface" bullying) and empathy, or between victimization and aggressiveness (either impulsive or premeditated). Furthermore, regression analysis revealed two predictors of victimization: low self-esteem and lower age.

Key words: Bullying, self-esteem, empathy, aggressiveness.

Correspondencia: Maite Garaigordobil. Facultad de Psicología. Universidad del País Vasco. Avda. de Tolosa, 70. C.P. 20018. Donostia-San Sebastián (España). E-mail: maite.garaigordobil@ehu.es 
Los problemas de convivencia escolar están muy presentes en las aulas, y no sólo dificultan el desarrollo de los procesos de enseñanza-aprendizaje, sino que además tienen consecuencias nefastas para el desarrollo humano (Garaigordobil y Oñederra, 2009, 2010a; Garaigordobil, 2011abc). La alarma social surge precisamente cuando se evidencian los nocivos efectos del maltrato para todos los implicados. En el peor de los casos las víctimas perecen ante el miedo y el dolor quitándose la vida.

Lamentablemente, el bullying es un tema vigente, y constituye una fuente de gran preocupación social. Todo ello pone de relieve la necesidad de llevar a cabo investigaciones que aporten conocimientos sobre las víctimas, especialmente sobre las conexiones entre victimización, y otras variables como empatía, autoestima, agresividad. Los resultados aportarán información de utilidad de cara al desarrollo de programas de intervención para prevenir la violencia entre iguales.

Victimización: Diferencias en función del género y el nivel socio-económicocultural

La revisión de la literatura que estudia las diferencias de género en victimización arroja resultados inconsistentes. La mayor parte de los estudios señalan que los varones son más víctimas de acoso que las mujeres (Ayenibiowo y Akinbode, 2011; Carlerby, Viitasara, Knutsson, y Gadin, 2012; Cerezo y Ato, 2010; Marées y Petermann, 2010; Scheithauer, Hayer, Petermann y Jugert, 2006).

Por el contrario, otras investigaciones ponen de relieve que las mujeres son más víctimas de acoso que los varones (Borg, 1999; Whitney y Smith, 1993), mientras que otros estudios no hallan diferencias de género en victimización (Card, Stucky, Sawalani, y Little, 2008; Chen y Astor, 2009; Peskin, Tortolero y Markham, 2006; Walden y Beran, 2010).

Los estudios que han analizado diferencias en victimización en función del nivel socio-económico-cultural familiar (NSEC) señalan que los participantes pertenecientes a familias con NSEC bajo fueron más víctimas (Cerezo y Ato, 2010; Davis et al., 2008). Otros estudios han encontrado que los agresores también pertenecían a familias con poco poder adquisitivo (Carlerby et al., 2012; Cerezo y Ato, 2010). En esta dirección Li, Lynch, Kalvin, Liu y Lerner (2011) hallaron que los adolescentes que pertenecían a familias con NSEC alto se involucraban con menor frecuencia en conductas de acoso, sin embargo, otros estudios no encuentran relación entre acoso y nivel socioeconómico familiar (Barboza et al., 2009). Los estudios que han investigado las diferencias en función del NSEC del centro educativo confirman que en los centros con NSEC bajo hay mayor incidencia de conductas de acoso, tanto de victimización como de agresión (Peskin et al., 2006). 
Victimización: relaciones con autoestima, empatía y agresividad impulsiva y premeditada

Los estudios que analizan la relaciones existentes entre victimización y otras variables como autoestima, empatía, y agresividad constatan que las víctimas tienen menor autoestima que aquellos que no han sufrido episodios de bullying (Estévez, Murgui y Musitu, 2009; Garaigordobil, 2011c; Garaigordobil y Oñederra, 2010a; Jankauskiene, Kardelis, Sukys, y Kardeliene, 2008; McMahon, Reulbach, Keeley, Perry, y Arensman, 2010; Suresh y Tipandjan, 2012). Además hay estudios que afirman que las víctimas tienen menor empatía que las no víctimas, que la victimización está fuertemente relacionada con bajos niveles de empatía (Malti, Perren, y Buchmann, 2010; Menesini, Camodeca, y Nocentini, 2010; Wang, Chen, Xiao, Ma y Zhang, 2011; Warden y Mackinnon, 2003).

En cuanto a la relación entre victimización y agresividad, hay estudios que afirman que las víctimas en general tienden en mayor medida que las no víctimas a ejercer conductas violentas (Turkel, 2007), sin embargo los estudios que diferencian entre víctimas y víctimas/agresoras, afirman que son estas últimas las que son más agresivas y no las víctimas puras (Perren y Alsaker, 2006; Ragatz, Anderson, Fremow, y Schwartz, 2011). Finalmente un meta-análisis llevado a cabo con 7 estudios afirma que es la agresividad reactiva, y no la proactiva, la que se relaciona con la victimización (Card y Little, 2006).

\section{Objetivos e hipótesis del estudio}

Tomando como base las investigaciones previas, el presente estudio tuvo 3 objetivos: 1) Analizar si existen diferencias en función del género y el nivel socioeconómico-cultural (NSEC) en victimización; 2) Estudiar las relaciones entre victimización y otras variables como autoestima, empatía, y agresividad, explorando si existen diferencias entre las víctimas y las no-víctimas en estas variables; y 3) Identificar variables predictoras de victimización, de ser víctima de bullying "cara a cara". Con esta contextualización se proponen 3 hipótesis: 1) Recientemente se está planteando la hipótesis de la similitud de género que defiende la semejanza entre varones y mujeres. Esta hipótesis emerge debido a los cambios que se han producido en la realidad social en los últimos años y que han puesto de relieve más similitud entre ambos sexos que en años precedentes. Por esta razón la hipótesis 1 del estudio plantea que no se encontrarán diferencias de género en victimización; 2) Teniendo en cuenta los estudios previos se propone que los y las adolescentes de un NSEC bajo sufrirán mayor victimización, en comparación con los y las adolescentes de NSEC alto; y 3) Tomando en consideración los resultados de algunos estudios correlacionales y cuasiexperimentales se postula que las víctimas tendrán un nivel más bajo de autoestima, menor empatía y mayor agresividad, siendo la baja autoestima un predictor de victimización. 


\section{METODO}

\section{Participantes}

La muestra está constituida por 178 adolescentes, de 13-15 años, que cursan Educación Secundaria Obligatoria (ESO), y están inscritos en 3 centros educativos de la ciudad de Donostia-San Sebastián (Gipuzkoa). Del conjunto de la muestra 78 son varones $(43.8 \%)$ y 100 mujeres $(56.2 \%)$. Para la selección de la muestra se utilizó una técnica de muestreo aleatorio simple, teniendo en cuenta tres niveles socio-económicoculturales (bajo, medio, alto). Con los centros estratificados, se seleccionó aleatoriamente un centro en cada estrato: (1) nivel bajo, familias humildes, con poca formación cultural $(N=77 ; 43.2 \%)$; (2) nivel medio, con formación cultural diversa $(N=37 ; 20.7 \%)$; y (3) nivel alto, culturalmente bien formados $(N=59 ; 33.1 \%)$.

\section{Instrumentos de evaluación}

Con la finalidad de evaluar las variables objeto de este estudio se administraron 3 instrumentos de evaluación con garantías psicométricas de fiabilidad y validez.

AVE. Acoso y Violencia Escolar (Piñuel y Oñate, 2006). Evalúa el acoso escolar o bullying, explorando un amplio rango de comportamientos de violencia psicológica, que a diferencia de la violencia física, deja pocas huellas externas, aunque es causa de inefables sufrimientos y perdurables daños psicológicos. A pesar de que el AVE desarrolla 2 dimensiones de acoso escolar (índice global de acoso e intensidad del acoso) para este estudio se han empleado únicamente los ítems que evalúan el índice global de acoso (IGA) que hace referencia a la frecuencia de diferentes conductas de hostigamiento psicológico. Esta dimensión está compuesta por 50 ítems, y el adolescente debe informar la frecuencia con la que le ocurre lo que indica la frase (nunca, pocas veces, muchas veces). Las afirmaciones hacen referencia a conductas de hostigamiento, de intimidación, amenazas a la integridad, coacciones, bloqueo o exclusión social, conductas directas de agresión ya sea física o psicológica. Por ejemplo, "roban mis cosas" "rompen mis cosas a propósito" "se ríen de mi" "me amenazan con armas" "no me dejan participar, me excluyen" "me zarandean o empujan para intimidarme". El coeficiente de fiabilidad se calculó mediante el alfa de Cronbach evidenciando una consistencia interna elevada $(\alpha=.95)$, y el coeficiente obtenido con la muestra de este estudio apunta en la misma dirección $(\alpha=.91)$. Para la validez estructural se realizó un análisis de componentes principales con rotación oblicua, indicando buen ajuste. Las saturaciones factoriales de cada elemento fueron superiores a .25.

RSE. Escala de autoestima (Rosenberg, 1965). Esta escala evalúa la autoestima general con 10 afirmaciones que aluden a sentimientos globales de autovaloración (en general estoy satisfecho conmigo mismo), 5 de los cuales están 
redactados en sentido positivo y 5 en sentido negativo. El sujeto debe leer las afirmaciones e informar en qué medida pueden ser aplicadas a él mismo, haciendo la valoración sobre una escala de tipo Likert con 4 categorías de respuesta (desde muy de acuerdo, a muy en desacuerdo). Por ejemplo, "creo que tengo varias cualidades buenas" "A veces me siento verdaderamente inútil". La fiabilidad de la prueba ha sido ampliamente documentada en la literatura. McCarthy y Hoge (1982) han informado de coeficientes de consistencia (alpha de Cronbach) que se sitúan entre .74 y .77, y de fiabilidad test-retest de .63 (intervalo de 7 meses) y de .85 (intervalo de 2 semanas). En la misma dirección el coeficiente alpha de Cronbach obtenido con la muestra de este estudio es alto $(\alpha=.88)$. La validez de la escala como medida unidimensional de la autoestima ha sido también comprobada en varios estudios (Silber y Tippett, 1965).

IECA. Índice de Empatía para niños y adolescentes (Bryant, 1982). Evalúa la activación empática de los adolescentes a través de 22 afirmaciones, algunas de las cuales reflejan situaciones en las que se da una activación empática hacia otra persona ante una situación determinada, mientas que otras reflejan una falta de empatía. Por ejemplo, "Me gusta ver como otras personas abren regalos aunque yo no tenga ningún regalo" "Soy capaz de comerme todas las galletas aunque vea que alguien me está mirando y quiere una". La escala evalúa 2 dimensiones: por un lado, la empatía afectiva, entendida como una activación emocional que hace referencia a mecanismos mínimamente cognitivos; y, por otro lado, las creencias sobre la expresión de sentimientos. El adolescente puntúa cada frase en una escala de 1 a 7 en función del grado de acuerdo con el contenido de la frase. La consistencia interna de la escala original obtuvo un alpha de Cronbach de .68 en cuarto grado y .79 en séptimo grado, mientras que la obtenida con la muestra de este estudio fue más alta $(\alpha=.83)$. Un estudio de fiabilidad test-retest con 108 participantes evidenció un coeficiente de correlación .81 con la muestra de cuarto grado y de .83 con estudiantes de séptimo. Estudios de validez han encontrado correlaciones positivas con otras medidas de empatía y negativas con conducta antisocial (Sobral, Romero, Luengo, y Marzoa, 2000) y conducta violenta (de Wied, Goudena, y Matthys, 2005).

CAPI-A. Cuestionario de agresividad premeditada e impulsiva en adolescentes (Andreu, 2010). La agresividad muestra diferentes formas de expresión: física o psicológica, activa o pasiva, directa o indirecta,... El CAPI-A evalúa la agresividad premeditada (medio instrumentalizado dirigido a la obtención de un objetivo diferente a dañar a la víctima) y la agresividad impulsiva (hace referencia a una respuesta no planificada, derivada de la ira y basada en la motivación de dañar a la víctima, como resultado de la provocación percibida). Se compone de 24 afirmaciones que deben leer e informar el grado de acuerdo con el contenido de la frase en una escala Likert de 1 a 5 (de muy en desacuerdo a muy de acuerdo). Por ejemplo, "creo que mi agresividad está justificada" "cuando me pongo furioso, reacciono sin pensar" "ser 
agresivo me ha permitido tener poder sobre los demás y mejorar mi nivel social". Los estudios psicométricos confirman la fiabilidad de la escalas de agresividad premeditada $(\alpha=.83)$ e impulsiva $(\alpha=.82)$. Los coeficientes obtenidos con la muestra de este estudio apuntan en la misma dirección $(\alpha=82)$. El CAPI-A correlacionó con el RPQ (Andreu, Peña, y Ramírez, 2009) confirmándose correlaciones positivas con impulsividad y con agresividad reactiva/proactiva, lo que confirma su validez convergente. La agresividad premeditada presentó una correlación ligeramente más elevada con agresividad proactiva y la agresividad impulsiva mostró mayor correlación con la agresividad reactiva.

\section{Diseño y procedimiento}

El estudio utilizó una metodología descriptiva, comparativa y correlacional. Primero se contactó con los directores de los centros educativos seleccionados, donde se les explicaron los objetivos del estudio, y se solicitó la autorización del centro para la realización de la investigación. Después se solicitó el consentimiento informado a padres y a adolescentes, procediendo a la administración de los instrumentos de evaluación en el aula, de forma colectiva, y en una sesión de evaluación que fue realizada por dos investigadoras del equipo. El estudio cumplió con los valores éticos requeridos en la investigación con seres humanos (consentimiento informado y derecho a la información, protección de datos y confidencialidad, gratuidad, no discriminación, y posibilidad de abandonar el estudio en cualquiera de sus fases), habiendo recibido la evaluación favorable del comité de ética de la Universidad del País Vasco.

\section{RESULTADOS}

Victimización: diferencias en función del género y el nivel socio-económico cultural

Con el objetivo de analizar diferencias de género en victimización, así como diferencias en función del NSEC, después de realizar la prueba de Levene para verificar el supuesto de homocedasticidad encontrando igualdad de varianzas, se calcularon las medias, desviaciones típicas y se realizó un análisis de varianza univariante. Los resultados confirmaron que aunque las mujeres tuvieron puntuaciones superiores en victimización $(M=4.93 ; D T=7.20)$ en comparación con los varones $(M=3.41$; $D T=4.42)$, las diferencias no fueron estadísticamente significativas, $F_{(1,176)}=2.68$; $p=.103$, siendo pequeño el tamaño del efecto $(d$ de Cohen=.25). Por lo tanto, el nivel de victimización en varones y mujeres fue similar. Por otro lado, los resultados de los análisis que exploran diferencias en función del NSEC (bajo, medio y alto), muestran que en victimización, aunque los participantes inscritos en el nivel alto $(M=3.60$; $D T=5.07)$ tiene menor puntuación que aquellos de niveles bajo $(M=4.60 ; D T=7.15)$ y medio $(M=4.61 ; D T=5.56)$, las diferencias no son estadísticamente significativas, 
$F_{(2,175)}=0.52 ; p=.595$, siendo muy pequeño el tamaño del efecto $\left(\eta^{2}=.006, r=.07\right)$. Además, los resultados de la prueba de comparación de grupos de Bonferroni ratificó la ausencia de diferencias significativas entre los tres niveles. Por lo tanto, el nivel de victimización en los distintos niveles socioeconómicos y culturales fue similar.

Victimización: conexiones con autoestima, empatía y agresividad

En primer lugar, con la finalidad de analizar las relaciones existentes entre victimización, y variables como autoestima, empatía, y agresividad, se obtuvieron los coeficientes de correlación de Pearson entre estas variables, con la muestra de varones y mujeres de forma diferenciada. Los resultados obtenidos se presentan en la tabla 1.

\begin{tabular}{|c|c|c|}
\hline & \multicolumn{2}{|c|}{ Victimización } \\
\hline & Varones $(N=78)$ & Mujeres $(N=100)$ \\
\hline Autoestima & $-.22 *$ & $-.27 * * *$ \\
\hline Empatía & .02 & .13 \\
\hline Agresividad Premeditada & .09 & .06 \\
\hline Agresividad Impulsiva & .16 & .15 \\
\hline
\end{tabular}

Tal y como se puede observar en la tabla 1, los coeficientes de correlación de Pearson ponen de relieve correlaciones negativas significativas entre victimización y autoestima, en la muestra de varones y mujeres. Sin embargo, no se encuentran correlaciones significativas entre victimización y variables como empatía y agresividad. Por consiguiente, los resultados sugieren que los adolescentes de ambos sexos que tuvieron altas puntuaciones en victimización tenían significativamente menor nivel de autoestima. Sin embargo, no se hallaron correlaciones significativas entre victimización y variables como empatía y agresividad ni premeditada ni impulsiva.

Complementariamente, con la finalidad de ratificar estos resultados, se dividió la muestra en dos perfiles, aquellos que tenían altas puntuaciones en victimización (percentil 85-99) y aquellos con puntuaciones inferiores a este punto de corte, procediendo a comparar ambos grupos. Para ello se realizó un MANOVA con las puntuaciones obtenidas en todas las variables (autoestima, empatía, agresividad), cuyos resultados no evidenciaron diferencias significativas entre ambos grupos, Lambda de Wilks, $\Lambda=.953 ; F_{(4,169)}=2.91 ; p>.05$, siendo el tamaño del efecto pequeño, $\eta^{2}=.047$, $r=.21$. Posteriormente, después de realizar la prueba de Levene para verificar el supuesto de homocedasticidad encontrando igualdad de varianzas, se calcularon las medias, desviaciones típicas y se realizó un ANOVA en cada variable evaluada, cuyos resultados se presentan en la tabla 2. 
Tabla 2. Medias, desviaciones típicas, resultados del análisis de varianza y tamaño del efecto (d de Cohen) entre víctimas y no víctimas en las variables objeto de estudio

\begin{tabular}{lcrrrrr}
\hline & \multicolumn{1}{c}{ No-Víctimas $(N=154)$} & \multicolumn{2}{c}{ Víctimas $(N=24)$} & \multirow{2}{*}{$F_{(1,176)}$} & \multirow{2}{*}{$d$} \\
\cline { 2 - 5 } & \multicolumn{1}{c}{$M$} & \multicolumn{1}{c}{$D$} & \multicolumn{1}{c}{$D$} & & \\
\hline Autoestima & 31.66 & 6.37 & 28.62 & 5.90 & $4.77^{*}$ & .49 \\
Empatía & 95.75 & 19.7 & 103.75 & 19.80 & 3.40 & -.40 \\
Agresividad Premeditada & 27.35 & 7.21 & 27.25 & 7.57 & 0.00 & .01 \\
Agresividad Impulsiva & 30.93 & 7.21 & 33.29 & 11.56 & 1.25 & -.24 \\
\hline Nota: ${ }^{*} p<.05$ & & & & & &
\end{tabular}

Tal y como se puede observar en la tabla 2, se ratifica que las víctimas tenían puntuaciones significativamente inferiores en autoestima. Sin embargo, no se hallaron diferencias en empatía y en agresividad, ni premeditada ni impulsiva.

\section{Variables predictoras de victimización}

Para identificar las variables que predicen victimización se realizó un análisis de regresión lineal múltiple, método de pasos sucesivos, cuyos resultados se presentan en la tabla 3. En este análisis se introdujeron las siguientes variables: sexo, edad, nivel socio-económico-cultural, autoestima, empatía, agresividad impulsiva y premeditada. Los resultados obtenidos se exponen en la tabla 3.

\begin{tabular}{lcccccccr}
\multicolumn{7}{c}{ Tabla 3. Variables predictoras de victimización } \\
\hline & $R$ & $R^{2}$ & $\Delta R^{2}$ & $B$ & Error típico & Constante & $\beta$ & \multicolumn{1}{c}{$t$} \\
\hline Autoestima & .26 & .07 & .06 & -.26 & 6.02 & 12.36 & -.269 & $-3.69^{* * *}$ \\
Edad & .31 & .09 & .08 & -1.24 & 5.95 & 29.95 & -.163 & $-2.24^{*}$ \\
\hline Nota: ${ }^{*} p<.05$ & $* *$ & $p<.01$ & $* * * p<.001$ & & & &
\end{tabular}

Del conjunto de variables predictoras de victimización (ver tabla 3), dos resultaron significativas: autoestima y edad. Los coeficientes de regresión estandarizados Beta indican que estas variables tienen poco peso sobre la variable criterio, la victimización. Los porcentajes de varianza explicada (coeficientes de determinación ajustados) por cada una de tales variables predictoras fueron de magnitud baja. Dos variables, que explican el $8 \%$ de la varianza, resultaron predictoras de victimización: baja autoestima y menor nivel de edad.

\section{DISCUSIÓN}

Este estudio tuvo como objetivos analizar si existen diferencias en función del género y el nivel socio-económico-cultural en victimización, estudiar las relaciones entre victimización y otras variables (autoestima, empatía, agresividad) e identificar variables predictoras de victimización. En primer lugar, los resultados obtenidos confirman la ausencia de diferencias de género en victimización. Por consiguiente, se ratifica la hipótesis 1. Estos resultados apuntan en la misma dirección que otros estudios que no 
han hallado diferencias de género en victimización (Card et al., 2008; Chen y Astor, 2009; Peskin et al., 2006; Walden y Beran, 2010), y apoyan la hipótesis de similitud de género al menos en lo que se refiere a victimización. No obstante, contradicen los resultados de aquellos estudios que han encontrado que los varones son más víctimas de bullying (Ayenibiowo y Akinbode, 2011; Carlerby et al., 2012; Cerezo y Ato, 2010; Marées y Petermann, 2010), o que las mujeres son más víctimas (Borg, 1999; Whitney y Smith, 1993). Las discrepancias pueden ser explicadas por las diferentes edades de las muestras de los estudios y los instrumentos de evaluación utilizados.

En segundo lugar, los resultados muestran que en victimización no hay diferencias en función del NSEC. Por lo tanto, se refuta la hipótesis 2 ya que proponía que los de NSEC bajo sufrirían mayor nivel de victimización. Estos resultados confirman los obtenidos en otros estudios que no han encontrado relación entre acoso y nivel socioeconómico familiar (Barboza et al., 2009), pero difieren de aquellos que han hallado en el nivel NSEC bajo, mayor nivel de victimización (Cerezo y Ato, 2010; Davis et al., 2008), y de implicación en situaciones de bullying (Li et al., 2011). Quizás las diferencias puedan explicarse por las características de la muestra, ya que en algunos estudios la muestra de nivel bajo está configurada por participantes inscritos en un nivel muy bajo (situaciones socioeconómicas muy desfavorecidas, de gran precariedad y vulnerabilidad), mientras que el nivel bajo de la muestra de este estudio no es tan extremo.

Finalmente, los coeficientes de correlación han evidenciado que los adolescentes de ambos sexos con altas puntuaciones en victimización tienen significativamente menor nivel de autoestima. Sin embargo, no hay diferencias entre víctimas y no víctimas en empatía ni en agresividad (ni impulsiva, ni premeditada). Además, en el análisis de regresión dos variables resultaron predictoras de victimización: baja autoestima y menor nivel de edad. Estos resultados confirman parcialmente la hipótesis 3, ya que las víctimas tienen menor autoestima, pero tienen similar nivel de empatía y de agresividad que las no víctimas. Estos resultados confirman los obtenidos en otros estudios que han encontrado menor nivel de autoestima en las víctimas (Estévez et al., 2009; Garaigordobil, 2011c; Garaigordobil y Oñederra, 2010a; Jankauskiene et al., 2008; McMahon et al., 2010; Suresh y Tipandjan, 2012). Además, los resultados confirman los obtenidos por aquellos que diferenciando entre víctimas y víctimas/agresoras, afirman que son estas últimas las que son más agresivas y no las víctimas puras (Perren y Alsaker, 2006; Ragatz et al., 2011). Sin embargo, los hallazgos de esta investigación contradicen los resultados obtenidos en otros estudios que han encontrado menor empatía en las víctimas (Malti et al., 2010; Menesini et al., 2010; Wang et al., 2011). Quizás esta discrepancia pueda explicarse por la edad de las muestras de estos estudios que están realizados con estudiantes de primaria, a diferencia de este estudio que ha sido llevado a cabo con adolescentes. 
Los hallazgos de la investigación implican una aportación a este ámbito de conocimiento y tienen implicaciones prácticas relacionadas con la prevención del bullying, ya que el trabajo ha permitido identificar objetivos de intervención. Los datos sugieren la necesidad de implementar programas de intervención psicoeducativa en la infancia y en la adolescencia para mejorar la convivencia y prevenir la violencia. Teniendo en cuenta los hallazgos del estudio, estos programas deben contener actividades que fomenten la autoestima de todos los miembros del grupo y, especialmente, de las víctimas. Tal y como se ha puesto de relieve en diversos estudios, la mejor forma de prevenir la violencia es construir la convivencia (Garaigordobil, 2011d), y para ello las personas deben sentirse seguras, dignas de ser queridas y valiosas, es decir, deben tener una positiva valoración de sí mismas.

Como limitaciones de este estudio cabe señalar el estrecho rango de edades de la muestra (13-15 años), y el uso de autoinformes con el sesgo de deseabilidad que conllevan. Como línea futura de investigación cabe sugerir la utilización de metodología experimental para ratificar los hallazgos de este estudio. Por ejemplo, aplicando programas que fomenten la autoestima, y midiendo el efecto en la disminución de conductas de victimización. La comparación del cambio pretest-postest en los participantes experimentales y control, permitirá evidenciar los nexos causales existentes entre estas variables y la eficacia de la intervención. Para futuros estudios también se puede sugerir ampliar el rango de edad de la muestra (10-18 años), así como el espectro muestral de niveles NSEC que incluyan niveles extremos (muy bajo o muy alto).

Agradecimientos: Estudio financiado por el Ministerio de Economía y Competitividad (MINECO) (PSI2012-30956), por el Departamento de Educación, Universidades e Investigación del Gobierno Vasco (IT638-13), y por la Unidad de Formación e Investigación de la Universidad del País Vasco UPV/EHU (UFI PSIXXI 11/04).

\section{REFERENCIAS}

Andreu, J.M. (2010). CAPI-A: Cuestionario de Agresividad Premeditada e Impulsiva en Adolescentes. Madrid: TEA.

Andreu, J.M., Peña, M.E. y Ramírez, J.M. (2009). Cuestionario de agresión reactiva y proactiva un instrumento de medida de la agresión en adolescentes. Revista de Psicopatología y Psicología Clínica, 14(1), 37-49.

Ayenibiowo, K.O. y Akinbode, G.A. (2011). Psychopathology of bullying and emotional abuse among school children. IFE Psychologia: An International Journal, 19(2), 127-141.

Barboza, G., Schiamberg, L., Oehmke, J., Korzeniewski, S., Post, L. y Heraux, C. (2009). Individual Characteristics and the Multiple Contexts of Adolescent Bullying: An Ecological Perspective. Journal of Youth and Adolescence, 38(1), 101-121.

Borg, M.K. (1999). The extent and nature of bullying among primary and secondary school children. Educational Research, 41(2), 137-153. 
Bryant, B.K. (1982). An index of empathy for children and adolescents. Child Development, 53, 413-425.

Card, N.A. y Little, T.D. (2006). Proactive and reactive aggression in childhood and adolescence: A meta-analysis of differential relations with psychosocial adjustment. International Journal of Behavioral Development, 30(5), 466-480.

Card, N.A., Stucky, B.D., Sawalani, G.M. y Little, T.D. (2008). Direct and indirect aggression during childhood and adolescence: A meta-analytic review of gender differences, intercorrelations, and relations to maladjustment. Child Development, 79(5), 1185-1229.

Carlerby, H., Viitasara, E., Knutsson, A. y Gadin, K. (2012). How bullying involvement is associated with the distribution of parental background and with subjective health complaints among Swedish boys and girls. Social Indicators Research, (preprints), 1-9.

Cerezo, F. y Ato, M. (2010). Social status, gender, classroom climate and bullying among adolescents pupils. Anales de Psicología, 26(1), 137-144.

Chen, J. y Astor, R. (2009). The perpetration of school violence in Taiwan. An analysis of gender, grade level and school type. School Psychology International, 30(6), 568-584.

Davis, E., Davies, B., Cook, K., Waters, E., Gibbs, L. y Priest, N. (2008). A Cross-Cultural Examination of Barriers to Social Inclusion for Children: A Qualitative Study Using Child-Centred Methods. International Journal of Mental Health Promotion, 10(1), 4252.

De Wied, M., Goudena, P. y Matthys, W. (2005). Empathy in boys with disruptive behavior disorders. Journal of Child Psychology and Psychiatry, and Aplied Disciplines, 46(8), 867-880.

Estévez, E., Murgui, S. y Musitu, G. (2009). Psychological adjustment in bullies and victims of school violence. European Journal of Psychology of Education, 24(4), 473-483.

Garaigordobil, M. (2011a). Bullying y cyberbullying: conceptualización, prevalencia y evaluación. En FOCAD Formación Continuada a Distancia. Duodécima Edición Enero-Abril 2011 (pp. 1-22). Madrid: Consejo General de Colegios Oficiales de Psicólogos.

Garaigordobil, M. (2011b). Bullying y cyberbullying: programas y estrategias de prevención e intervención desde el contexto escolar y familiar. En FOCAD Formación Continuada a Distancia. Duodécima Edición Enero-Abril 2011 (pp. 1-29). Madrid: Consejo General de Colegios Oficiales de Psicólogos.

Garaigordobil, M. (2011c). Prevalencia y consecuencias del cyberbullying: Una revisión. International Journal of Psychology and Psychological Therapy, 11(2), 233-254.

Garaigordobil, M. (2011d). Entrevista: Jugar y cooperar contra el acoso escolar. Cuadernos de Pedagogía, 417(noviembre), 44-49.

Garaigordobil, M., y Oñederra, J.A. (2009). Acoso y violencia escolar en la comunidad autónoma del País Vasco. Psicothema, 21(1), 83-89.

Garaigordobil, M. y Oñederra J.A. (2010a). La Violencia entre iguales: Revisión teórica y estrategias de intervención. Madrid: Pirámide.

Garaigordobil, M. y Oñederra J. A. (2010b). La mejor forma de prevenir la violencia es construir la convivencia. Comunidad Escolar. Periódico digital de información educativa, 877. Ministerio Educación. Gobierno de España. http://comunidadescolar.educacion.es/877/entrev.html

Jankauskiene, R., Kardelis, K., Sukys, S. y Kardeliene, L. (2008). Associations between school bullying and psychosocial factors. Social Behavior and Personality, 36(2), 145-162.

Li, Y., Lynch, A., Kalvin, C., Liu, J. y Lerner, R. (2011). Peer relationships as a context for the development of school engagement during early adolescence. International Journal of Behavioral Development, 35(4), 329-342. 
Malti, T., Perren, S. y Buchmann, M. (2010). Children's peer victimization, empathy, and emotional symptoms. Child Psychiatry and Human Development, 41(1), 98-113.

Marées, N. y Petermann, F. (2010). Bullying in German primary schools: Gender differences, age trends and influence of parents' migration and educational backgrounds. School Psychology International, 31(2) 178-198.

McCarthy, J.D. y Hoge, D.R. (1982). Analysis of age effects in longitudinal studies of adolescent self-esteem. Developmental Psychology, 18, 372-379.

McMahon, E., Reulbach, U., Keeley, H., Perry, I. y Arensman, E. (2010). Bullying victimisation, self harm and associated factors in Irish adolescent boys. Social Science and Medicine, $71,1300-1307$.

Menesini, E., Camodeca, M. y Nocentini, A. (2010). Bullying among siblings: The role of personality and relational variables. British Journal of Developmental Psychology, 28(4), 921-939.

Perren, S. y Alsaker, F.D. (2006). Social behavior and peer relationships of victims, bully-victims, and bullies in kindergarten. Journal of Child Psychology and Psychiatry, 47(1), 45-57.

Peskin, M., Tortolero, S. y Markham, C. (2006). Bullying and victimization among black and hispanic adolescents. Adolescence, 41(163), 467-484.

Piñuel, I. y Oñate, A. (2006). AVE: Acoso y Violencia Escolar. Madrid: TEA.

Ragatz, L.L., Anderson, R.J., Fremouw, W. y Schwartz, R. (2011). Criminal thinking patterns, aggression styles, and the psychopathic traits of late high school bullies and bullyvictims. Aggressive Behavior, 37(2), 145-160.

Rosenberg, M. (1965). Society and the adolescent self-image. Princeton, NJ: Princeton University Press.

Scheithauer, H., Hayer, T., Petermann, F. y Jugert, G. (2006). Physical, verbal, and relational forms of bullying among German students: age trends, gender differences, and correlates. Aggressive Behavior, 32(3), 261-275.

Silber, E. y Tippet, J.S. (1965). Self-esteem: Clinical assessment and measurement validation. Psychological Reports, 16, 1017-1071.

Sobral, J., Romero, E., Luengo, A. y Marzoa, J. (2000). Personalidad y conducta antisocial: amplificadores individuales de los efectos contextuales. Psicothema, 12, 661-670.

Suresh, S. y Tipandjan, A. (2012). School bullying victimization and college adjustment. Journal of the Indian Academy of Applied Psychology, 38(1), 68-73.

Turkel, A. (2007). Sugar and spice and puppy dogs' tails: The psychodynamics of bullying. Journal of the American Academy of Psychoanalysis \& Dynamic Psychiatry, 35(2), 243 258.

Walden, L. y Beran, T. (2010). Attachment quality and bullying behavior in school-aged youth. Canadian Journal of School Psychology, 25(1) 5-18.

Wang, F., Chen, J., Xiao, W., Ma, Y. y Zhang, M. (2011). Prevalence of peer violence and its relation with violence belief and related skills in primary students. Chinese Mental Health Journal, 25(6), 449-453.

Warden, D. y Mackinnon, S. (2003). Prosocial children, bullies and victims: An investigation of their sociometric status, empathy and social problem-solving strategies. British Journal of Developmental Psychology, 21(3), 367-385.

Whitney, I. y Smith, P. K. (1993). A survey of the nature and extent of bullying in junior/middle and secondary schools. Educational Research, 35(1), 3-25.

Recibido: 21 de febrero de 2013

Recepción Modificaciones: 8 de marzo de 2013

Aceptado: 17 de marzo de 2013 